\title{
Der Pianist Lang Lang - Held der Hochkultur oder kurzlebige Celebrity?
}

\section{Ulrike Zimmermann}

\section{Lang Lang: Die Erfolgsgeschichte}

Der chinesische Pianist Lang Lang, der 1982 in Shenyang geboren wurde, ist in der klassischen Musikszene ein internationales wie multimediales Phänomen. Seine musikalischen Schwerpunkte liegen in der Romantik, mit Aufführungen von Werken von Tschaikowski, Liszt, Schubert und Chopin machte er sich einen Namen. In jüngerer Zeit orientiert sich Lang Lang aber weg von großen, paradigmatischen Klavierstücken, die ein virtuoses Spiel erfordern, hin zu kleineren Stücken und Werkauszügen; sein Repertoire wird somit kleinteiliger und diversifizierter. ${ }^{1}$ Er unterhält enge Verbindungen nach Deutschland, und seine hiesige Fangemeinde ist groß. Im Frühsommer 2019 war Lang Lang mit einer Konzertreihe in Deutschland unterwegs, die von medialer Aufmerksamkeit und vielen Interviews begleitet wurde. Anfang Juni heiratete Lang Lang die Wiesbadener Pianistin Gina Alice Redlinger, worüber die deutsche Presse ausführlich berichtete. ${ }^{2}$ Nicht nur in China und Deutschland, auch in den Vereinigten Staaten und in Australien (wohin ihn ein Teil seiner aktuellen Tour führt) füllt Lang Lang zuverlässig Konzertsäle. ${ }^{3}$

Lang Langs Erfolg als Profimusiker ist unbestritten, seine mediale Präsenz ubiquitär. Seine Facebookseite erreicht über eine halbe Million Menschen, auf Instagram ist er mit über 300.000 Followern präsent und auf Twitter mit etwa 145.000. ${ }^{4}$

Doch eine große Musiker-Karriere, musikalische Begabung und Erfolg genügen nicht unbedingt, um mit Fug und Recht als Held bezeichnet zu werden. Sicherlich gehören außerordentliche Eigenschaften zumeist zu einer Heldenfigur dazu. Oft zeichnen sich Helden auch dadurch aus, dass sie über große Handlungsmacht, sogenannte Agency, verfügen und diese gegen Widerstände einsetzen. Agonalität ist damit ebenfalls ein häufiges Kennzeichen von Heldentum. Aber Heldentum ist nicht als intrinsische Eigenschaft oder habituelle Verhaltensweise einer Person zu definieren; ohne kulturelle Zuschreibungen, die inner-

\footnotetext{
Lang Lang: Piano Book. Deutsche Grammophon, CD/LP, 2019.

2 Lang Lang hat geheiratet, in: Der Spiegel, 4. Juni 2019, www.spiegel.de/panorama/leute/ land-lang-hat-geheiratet-seine-frau-kommt-aus-wiesbaden-a-1270787.html, 29. Juni 2019.

3 Ein Blick in das Ticketsystem Reservix zeigt, dass das Konzert in Freiburg am 13. März 2020 praktisch ausverkauft war; die Kartenpreise bewegten sich zwischen etwa 140 und 190 Euro (Stand Ende Juni 2019).

4 Siehe Facebook, „Lang Lang“, www.facebook.com/LangLangPiano/, Instagram „@langlangpiano“, www.instagram.com/langlangpiano/?igshid=199unxy2e5xz0 und Twitter „@lang_lang“, twitter.com/lang_lang?lang=de, alle Oktober 2019.
} 
halb einer Gesellschaft wirken und häufig Kristallisationspunkt von Bedürfnissen der Mitglieder dieser Gesellschaft sind, ist Heldentum nicht vorstellbar. Helden müssen allgemein bekannt sein, und ihre Geschichten müssen erzählt werden, wenn sie und ihre außergewöhnlichen Taten nicht einfach untergehen sollen. Helden werden damit zu Projektionsflächen, zu Folien, auf denen sich soziokulturelle Agenden abbilden können. ${ }^{5}$

Exzeptionalität, seine außerordentliche musikalische Begabung, ist eine Eigenschaft, die Lang Lang sicher attestiert werden kann. Besonders medial attraktiv macht ihn außerdem seine Position als Wanderer zwischen Kulturen - der chinesischen und der ,westlichen' als professioneller Vertreter klassischer westlicher Musik - und als Vermittler dieser Kulturen. Es entbehrt schließlich nicht einer gewissen Ironie, dass klassische Musik aus Europa in China gegenwärtig mehr kulturelles Prestige zu haben scheint als an ihren Ursprungsorten. Doch auch im Fall von Lang Lang lässt sich die Frage, ob durch seine Person die Musik, verandert ${ }^{\star}$ wird und durch das Element eines Fremden die „Konsolidierung des Eigenen" verfolgt wird, nicht endgültig beantworten. ${ }^{6}$

Im Folgenden soll diese Position des Pianisten näher untersucht werden, und es wird sich zeigen müssen, ob und inwieweit das Konzept eines Helden überhaupt auf Lang Lang passt. Als Alternative zum Heldenbegriff soll geprüft werden, ob der Begriff der Berühmtheit, als Lehnwort ,Celebrity', auf Lang Lang treffender anwendbar wäre, und wie sich - das wäre eine zentrale Frage - Helden von Berühmtheiten oder Celebrities unterscheiden. Wo sind die Trennlinien, und ist es überhaupt möglich und sinnvoll, diese scharf zu ziehen? Gibt es Verschiebungen, Verwerfungen und Widersprüche?

Lang Lang begann das Klavierspiel mit drei Jahren. Seine frühe Ausbildung erfüllt alle Voraussetzungen einer Wunderkind-Erzählung. Der quasi prädestinierte Held, der schon in seiner Kindheit seine ungewöhnliche Begabung beweist, ist ein Präfigurat, das sich bereits beim antiken Helden Herakles findet, der als Säugling Schlangen mit den bloßen Händen erwürgen konnte. Ein Wunderkind mag nicht ganz so spektakulär sein, wird aber ebenfalls als früh auserwählt wahrgenommen. Der Begriff ist im Deutschen seit dem späten 16. Jahrhundert belegt und wurde zunächst hauptsächlich für den Christusknaben verwendet, aber auch für ein Kind,

5 Zu Grundlagen des Heroischen siehe etwa Ralf von den Hoff u. a.: Helden - Heroisierungen - Heroismen. Transformationen und Konjunkturen von der Antike bis zur Moderne. Konzeptionelle Ausgangspunkte des Sonderforschungsbereichs 948, in: helden. heroes. héros. E-Journal zu Kulturen des Heroischen 1, 2013, S. 7-14, DOI: 10.6094/helden. heroes.heros./2013/01/03; zu Entstehungsprozessen im Besonderen Tobias Schlechtriemen: Konstitutionsprozesse heroischer Figuren, 7. Juni 2018, in: Ronald G. Asch u. a. (Hg.): Compendium heroicum, DOI: 10.6094/heroicum/konstitutionsprozesse.

6 Achim Aurnhammer / Barbara Korte: Einleitung, in: dies. (Hg.): Fremde Helden auf europäischen Bühnen (1600-1900) (Helden - Heroisierungen - Heroismen 5), Würzburg 2016, S. 9-19, hier S. 11. 
das Wunder tun kann. ${ }^{7}$ Immanuel Kant gebrauchte den Begriff in säkularer Bedeutung für den 1721 geborenen Christian Heinrich Heineken, ein Sprach- und Mathematikgenie, das schon mit vier Jahren starb. Die bekannteste Persönlichkeit, die als Wunderkind firmierte, ist wohl Wolfgang Amadeus Mozart. Im 19. Jahrhundert setzte sich der Begriff im allgemeinen Sprachgebrauch durch und bezieht sich heute vor allem, aber nicht ausschließlich, auf Musiker und Musikerinnen. Wunderkinder - der Begriff kommt in der Wissenschaft kaum vor, man würde dort eher von Hochbegabung sprechen - sind ein umstrittenes Phänomen: auf der einen Seite faszinieren sie die Öffentlichkeit, vor der sie präsentiert werden und von der sie letztlich leben (und dies gilt im 18. wie im 21. Jahrhundert), auf der anderen Seite wird ihre mediale und damit einhergehende ökonomische Ausbeutung kritisiert, seit es den Begriff gibt. Schließlich haben Wunderkinder oft wenig von ihrem Status außer Mühen, Einschränkungen und Anstrengung; Profiteure, selbst wenn sie aus gutem Willen agieren, sind zumeist ihre Eltern und Lehrer. ${ }^{8}$

Als Lang Lang fünf Jahre alt war, gewann er den Klavierwettbewerb seiner Heimatstadt Shenyang. Mit neun Jahren nahm er sein Musikstudium an der zentralen Musikhochschule in Peking auf, und nur zwei Jahre später gewann er seinen ersten internationalen Preis in Süddeutschland beim Internationalen Wettbewerb für Junge Pianisten in Ettlingen bei Karlsruhe. Von da an ging es mit Lang Langs Karriere immer nur bergauf. Während seines Studiums von 1997-2002 am Curtis Institute in Philadelphia/USA gelang ihm der Durchbruch, als er bei der Galaxy of Stars des Ravinia Festivals kurzfristig für André Watts einspringen durfte, um mit dem Chicago Symphony Orchestra das 1. Klavierkonzert von Tschaikowski zu spielen. Sein Solodebüt hatte Lang Lang 2003 in der Carnegie Hall in New York, die zu diesem Anlass ausverkauft war. Seither arbeitet Lang Lang international und mit führenden Dirigenten wie Sir Simon Rattle und Daniel Barenboim und eilt von einem Erfolg zum anderen. Bei den Feierlichkeiten zu Mozarts 250. Geburtstag spielte er in der Großen Halle des Volkes in Peking. Im Sommer des gleichen Jahres trug er zu den Eröffnungsfeierlichkeiten der Fußballweltmeisterschaft in Deutschland bei. Im Jahr 2008 war Lang Lang auf der Bregenzer Seebühne zur Eröffnung der Fußball-Europameisterschaft, wo er mit Christopher von Deylen spielte, in der deutschsprachigen elektronischen Popmusikszene besser bekannt als Schiller. Es zeigt sich, dass Lang Lang fast vom Anfang seiner Karriere nicht nur bei klassischen Konzerten des üblichen Formats auftrat, sondern zunehmend bei größeren, popu-

7 „wunderkind“, in: Deutsches Wörterbuch von Jacob und Wilhelm Grimm, 16 Bde. in 32 Teilbänden, Leipzig 1854-1961, Quellenverzeichnis, Leipzig 1971, Online-Version: www. woerterbuchnetz.de/cgi-bin/WBNetz/wbgui_py?sigle=DWB\&mode=Vernetzung\&lemid= GW28408\#XGW28408, 13. Oktober 2019. Ich danke Achim Aurnhammer für den Hinweis auf die lange Geschichte des Begriffs.

Der Psychologe Andrew Salomon hat 2012 in seinem Buch Weit vom Stamm: Wenn Kinder ganz anders als ibre Eltern sind Porträts von Persönlichkeiten versammelt, die bereits im Kindesalter im Zentrum öffentlicher Aufmerksamkeit standen, und darin unter anderem $\mathrm{Mu}$ sikerinnen und Musiker wie Yevgeny Kissin, Vanessa Mae und Lang Lang aufgenommen. 
lären Events. In China gilt das Gleiche: die Olympischen Sommerspiele in Peking 2008 hatten Lang Lang im Eröffnungsprogramm. Für das Titellied der Expo in Shanghai (2010) begleitete er Jackie Chan am Klavier. In Großbritannien erfreut er sich ebenfalls großer Bekanntheit: beim Konzert der BBC anlässlich des diamantenen Kronjubiläums Königin Elisabeths II. im Jahr 2012 vor dem Buckingham Palace in London wirkte er unter einem großen Staraufgebot mit. Im gleichen Jahr trug er die olympische Fackel ein Stück auf ihrem Weg nach London zur Eröffnung der Olympischen Spiele. 2012 war zugleich ein wichtiges Jahr für Lang Langs Verbindung zu Deutschland, denn für seine Verdienste um die deutsche Musikkultur und im Besonderen für das Schleswig-Holstein Musik Festival wurde ihm das Bundesverdienstkreuz verliehen. ${ }^{9}$

Schon seit 2004 ist Lang Lang Friedensbotschafter (Ambassador of Goodwill) des Kinderhilfswerks UNICEF, und seit 2013 auch Friedensbotschafter der Vereinten Nationen. Es dürfte bei dieser Aufzählung seiner Aktivitäten augenfällig sein, dass seine genuinen Fähigkeiten als Pianist bislang eher wenig zur Sprache kamen. Nicht nur anhand seiner Aktivitäten und Auftritte in Deutschland lässt sich eine Tendenz feststellen, die in Lang Langs Karriere immer mehr hervorsticht: Er bewegt sich hin zur Breitenwirkung wie auch zu populärer Musik und musikalischer Unterhaltung. In seine Laufbahn als professioneller Musiker integriert er einen Bildungsauftrag: Er versucht, Kinder weltweit für klassische Musik zu gewinnen. ${ }^{10}$

Seine Fangemeinde ist nicht nur international, sondern sie umfasst auch viele junge Menschen, während gleichzeitig die Beliebtheit von klassischer Musik gerade in Europa zu schwinden scheint. Als Bürger Chinas ist Lang Lang inzwischen zu einem exponierten internationalen Werbeträger für klassische westliche Musik geworden, deren Prestige in China - aber eben nicht nur dort - unter anderem durch seine Karriere und seine mediale Präsenz gefördert wird. Seine Klavierschule für Kinder (die seit 2016 auch in deutscher Sprache erhältlich ist) soll in seiner Heimat 40 Millionen Kinder zum Klavierlernen bewogen haben. Seine Kurzbiographie in seinen Accounts auf den sozialen Netzwerken fasst seine Mission zusammen: „Pianist. Mentor. Lifelong Student. U.N. Messenger for Peace. Traveler. I believe that classical music is for everyone!" ${ }^{11}$

9 Die offizielle Bezeichnung ist Verdienstkreuz am Bande des Verdienstordens der Bundesrepublik Deutschland. Es ist die einzige allgemeine Verdienstauszeichnung der Bundesrepublik und würdigt unter anderem besondere kulturelle Verdienste.

10 Zur Biographie und bisherigen Lebensleistung Lang Langs gibt es eine Reihe von Quellen. Für Grundlagen genügt sicher der Wikipedia-Eintrag: de.wikipedia.org/wiki/Lang_Lang, 30. Juni 2019. Auf der Seite seines Labels, der Deutschen Grammophon, sind die Informationen nicht mehr ganz aktuell: www.deutschegrammphon.com/de/artist/langlang. Lang Lang-Fans mögen zur Autobiographie greifen: Lang Lang mit David Ritz: Musik ist meine Sprache. Die Geschichte meines Lebens, Berlin 2008.

11 Zitiert aus Lang Langs Twitter-Profil: twitter.com/lang_lang?lang=de, 30. Juni 2019. 


\section{Der populäre Pianist: Klassik und Kommerz}

Während Lang Lang von der Sinnhaftigkeit, klassische Musik zu erlernen und zu praktizieren, offenbar überzeugt ist und dafür mit Verve wirbt, ist seine Fähigkeit als Konzertpianist eher umstritten. Die deutsche Kritik ist in der vergangenen Dekade mit Lang Lang häufig harsch umgegangen. Er ist sicher talentiert, und es ist letztlich dieses Talent zusammen mit einer intensiven Ausbildung, das ihn berühmt gemacht hat. Die Frage in Teilen seines Publikums, ob er nun wirklich ein Pianist von hoher Qualität sei, begegnet im Laufe seiner Karriere immer öfter. Ein abschließendes Urteil kann an dieser Stelle natürlich nicht gefällt werden; ein kurzer Blick in die Kritik sei dennoch erlaubt.

Die Musikkritik in Deutschland hat sich gerne und schon recht früh über Defizite in Lang Langs Spiel geäußert. Unter dem Titel „Der Fluch des Virtuosen“ äußerte zum Beispiel Christine Lemke-Mathwey im Tagesspiegel schon 2007 Zweifel an Lang Langs echter Musikalität und berichtet vom Pianisten, „[...] der sich mit einer Energie sondergleichen längst in die traurigen Gefilde internationaler Megastars katapultiert hat." ${ }^{212}$ Lang Lang, so war in der Überschrift zu lesen, entdecke Beethoven und verfehle sich selbst. Es ist im Subtext gerade seine Beliebtheit, die abfällig erwähnt wird. Der Subtext ist elitär, denn wer ein ,echter ${ }^{\circ}$ klassischer Musiker ist, kann wohl kaum so beliebt und (auch finanziell) so erfolgreich sein. Diese Abwertung gründet implizit auf der Idee des einsamen, verarmten, aber genialischen Ausnahmekünstlers, ein Klischee, dem Lang Lang nun so gar nicht entsprechen mag.

$\mathrm{Zu}$ Lang Langs 30. Geburtstag im Jahr 2012 schrieben viele deutsche Zeitungen über ihn und versuchten, das Geheimnis seines Erfolgs zu analysieren. In einem Artikel des Tagesspiegel vom 14. Juni 2012 sinniert Frederick Hanssen, dass Daniel Barenboim vermutlich weniger bekannt sei als Lang Lang, obwohl ein musikalisches Schwergewicht. „Von Lang Lang dagegen haben auch jene Zeitgenossen schon mal gehört, die nicht wissen, was ein Dominantseptakkord ist." Offenbar weckt er Interesse bei Menschen, die Musik mögen, aber nicht über eine klassische Ausbildung verfügen. Typischerweise wird gerade diese Breitenwirkung eher kritisch gesehen, und der Unterton des Artikels wirkt ohne Zweifel etwas snobistisch. Weiter heißt es: „Mit 30 Jahren hat Lang Lang alles erreicht, was sich im Entertainmentbusiness erreichen lässt. Seine CDs verkaufen sich wie geschnitten Brot, seine Gagen erreichen Spitzenwerte [...].“ Der Tagesspiegel fährt allerdings dann mit der Benennung eines zentralen Problems fort: „Nur sein Image in der Klassikszene macht ihm zu schaffen. Dass er über alle spieltechnischen Fragen erhaben ist, steht außer Frage. Doch seine affektierte Gestik bei Liveauftritten wirkt nicht gerade seriös, interpretatorisch bleibt er oft in pubertärer Ge-

12 Christine Lemke-Mathwey: Fluch des Virtuosen, in: Der Tagesspiegel, 22. Juni 2007, www. tagesspiegel.de/kultur/pianist-lang-lang-fluch-des-virtuosen/965750.html, 29. Juni 2019. 
fühlsduselei stecken. ${ }^{13}$ Hanssen eröffnet hier die Unterscheidungskategorie zwischen E- und U-Musik, ernsthaften und unterhaltenden Musikrichtungen, die in Deutschland nach wie vor stark voneinander abgegrenzt werden.

Großer kommerzieller Erfolg scheint im Fall von Lang Lang stets mit der Frage einherzugehen, ob er die nötige intellektuelle Reife für sein eigenes Repertoire hat - dass er es spielen kann, steht außer Frage. Bei einer Persönlichkeit mit einer so großen Affinität zu den sozialen Netzwerken lohnt ein genauerer Blick auf die Fangemeinde. Auch in Klassik-Internetforen scheiden sich ganz offensichtlich die Geister an Lang Lang; es gibt viele Diskussionsbeiträge zu der Frage, ob er denn nun gut sei oder nicht. Ein Nutzer stellt auf der Seite Pianostreet.com fest: „Lang Lang ist die moderne Version von Franz Liszt. Technische Brillanz mit Charisma und manchmal wirklich, wirklich schlechtem Geschmack“. Die direkte Antwort auf diesen Vergleich kommt offenbar von einem Liszt-Verehrer: „Das ist die schlimmste Beleidigung von Liszt, die ich je gesehen habe“. Verschiedene Stimmen monieren, dass zu viel emotionales Getue und Show in den Vorstellungen von Lang Lang eine zu große Rolle spielten. Es wird aber mindestens ebenso häufig sein technisches Können angemerkt. ${ }^{14}$ Es ist augenfällig, dass die Urteile doch einigermaßen differenziert sind und, auch wenn sie nicht von Fans Lang Langs kommen, ihn nicht als professionellen Musiker komplett abtun können. Wer ihn nicht mag, erkennt dennoch seine technische Fähigkeit an.

Nach längerer Pause wegen einer Sehnenscheidenentzündung, die sich Lang Lang beim Üben zugezogen hatte, war er 2019 wieder auf Tour, die mit Begeisterung bei seinen Fans, jedoch mit mehr Skepsis in der deutschen Presse erwartet wurde. Interviews mit Lang Lang erschienen in einer Vielzahl von Zeitungen und Zeitschriften; die Frankfurter Allgemeine Zeitung brachte gar eine Modestrecke mit Lang Lang heraus. ${ }^{15}$

Die neue CD, das Piano Book, erhielt eher mäßige Kritiken. Sie versammelt viel Klassik-Mainstream und daraus besonders Stücke, die von Klavierlernenden zum Üben genutzt werden. Im Piano Book bestätigt sich die Tendenz Lang Lang

13 Frederick Hanssen: Wunderkind Lang Lang: Der Elfenbeinharte, in: Der Tagesspiegel, 14. Juni 2012, www.tagesspiegel.de/gesellschaft/panorama/wunderkind-lang-lang-der-elfen beinharte/6745884.html, 30. Juni 2019.

Der Nutzer „stevensk“ schreibt: „Lang Lang is the modern version of Franz Liszt. Technical brilliance associated with charisma and sometimes really bad taste." Ein anderer, „thalbergmad“, antwortet darauf: „Just about the worst insult concerning Liszt I have ever seen [...].“ Es würde an dieser Stelle zu weit führen, ausführlich auf Forendiskussionen einzugehen. Die erwähnten Beiträge lassen sich unter Pianostreet.com (Is Lang Lang good or not, 2015, www.pianostreet.com/smf/index.php?topic=23971.0), weitere auf Talkclassical.com (Opinion on Lang Lang, 2014, www.talkclassical.com/30161-opinion-lang-lang. html) nachlesen (30. Juni 2019).

15 Marc Zitzmann (Text) und Gregor Hohenberg (Fotos): Lang Lang, in: Frankfurter Allgemeine Zeitung, 31. März 2019 (N.B. Das Ergebnis der Internetsuche wie auch alle Verweise auf diesen Artikel haben das korrekte Datum; der Artikel selbst hat unter seiner Überschrift den 31. März 2018 angegeben, was ein Fehler ist), www.faz.net/aktuell/stil/mode-design/ star-pianist-lang-lang-beim-mode-shooting-in-paris-16078839.html, 29. Juni 2019. 
hin zu gezielter Breitenwirkung mit Material, das weniger Virtuosität braucht, bei Laien aber vielleicht besser ankommt. Tobias Stosiek kommentiert in seiner Kritik des Albums auf BR Klassik: „Tatsächlich kommt beim Blick auf die Trackliste des Albums der hartnäckige Verdacht auf, dass hier nicht nur nostalgische Gefühle, sondern auch kommerzielle Interessen ein Wörtchen mitzureden hatten.“ Nun scheint dieser Verdacht bei einem Musiker wie Lang Lang nicht besonders überraschend, denn er - und die Deutsche Grammophon, bei der er unter Vertrag ist - möchte ja seine Aufnahmen verkaufen. Stosiek erkennt die Spieltechnik Lang Langs an und stellt auch fest, dass die Auswahl vielleicht Menschen zur Klassik bringt, die sonst ganz andere Musik mögen. „Nervig ist allerdings sein Hang zum Kitsch: viel Pedal, viel Rubato." ${ }^{16}$

Ein guter Pianist zu sein, reicht nicht zum Helden. Wenn Lang Lang nun ein Pianist mit deutlichen Schwächen ist - kann er dennoch ein Held sein, oder hat er zu viele Schwächen und lebt als Figur nurmehr von seiner medialen ÜberPräsenz? Ist diese Art von Kritik ausreichend, um den Status eines Helden grundsätzlich in Frage zu stellen, zumal es sich hier zumindest teilweise um geschmackbasierte Kritik handelt? Ronald G. Asch und Michael Butter merken in der Einleitung zu ihrem Band über Helden und Publika an: „Historische im Gegensatz zu rein fiktiven Helden müssen so angelegt sein, dass ihre ,Konstruktion“ eine Kritik, die auf nachweisbare Defizite im Charakter oder in den Taten [...] verweist, aufzufangen vermag." 17 Ist die Konstruktion der öffentlichen Figur Lang Lang in diesem Sinne stark genug? Nachweisbare Defizite in den Taten sind auf jeden Fall zu finden. Ihretwegen, und wegen der Art, in der sich der Starkult um ihn herum entwickelt, passt auf Lang Lang der Begriff der Celebrity womöglich besser als der des Helden. ${ }^{18}$ Beide Phänomene haben einige Aspekte gemeinsam, und die Grenzen zwischen ihnen erscheinen teilweise fließend. Gerade im 21. Jahrhundert, das immer wieder - zumindest für einige Weltgegenden - als postheroisch bezeichnet wird (etwa von Herfried Münkler), erscheint es lohnenswert, sich die Bruchlinien zwischen Held und Celebrity genauer anzusehen.

Das englische Nomen celebrity geht auf den lateinischen Begriff der celebritas zurück, der „Berühmtheit“ und „Prominenz“ bedeutete, gleichzeitig auch „zahl-

16 Tobias Stosiek: Lang Langs Piano Book. Zwischen Genie und Kommerz, in: BR-Klassik, 6. April 2019, www.br-klassik.de/aktuell/meinung/cd-kritik-lang-lang-piano-book-100.html, 30. Juni 2019.

17 Ronald G. Asch / Michael Butter: Verehrergemeinschaften und Regisseure des Charisma. Heroische Figuren und ihr Publikum. Einleitung, in: dies. (Hg.): Bewunderer, Verehrer, Zuschauer: Die Helden und ihr Publikum (Helden - Heroisierungen - Heroismen 2), Würzburg 2016, S. 9-21, hier S. 10.

18 Im Sinne von Maltby u. a. stehen als Annahme für diesen Artikel „low levels of celebrity worship“. Die Celebrity verfügt über „the perceived ability to entertain and capture our attention"; es geht nicht um fanatischen Fankult. Vgl. John Maltby u. a.: A Clinical Interpretation of Attitudes and Behaviours Associated with Celebrity Worship, in: Journal of Nervous \& Mental Disease 191.1, Februar 2003, S. 25-29, hier S. 26. 
reicher Besuch, Belebtheit" ${ }^{19}$ Celebrity ist als Lehnwort im Deutschen angekommen und seit 2006 im Duden verzeichnet. Es bezeichnet wie im Englischen eine „berühmte Person“; das Englische hat als zweite Bedeutung auch den „Zustand des Berühmtseins“, wobei der Begriff stark die Kurzlebigkeit und Flüchtigkeit dieses so benannten Ruhmes konnotiert. Diese Flüchtigkeit ist es eben, die in dem englischen Begriff stärker mitschwingt als im deutschen Wort „Berühmtheit“, weshalb der Anglizismus durchaus seine Berechtigung hat. Es geht hierbei nicht nur um die Veralltäglichung und Demokratisierung von Heldentum, sondern um einen Bedeutungsaspekt, der stärker auf dem Ruf und dem Ruhm liegt, also dem Teil von Heldentum, der sich aus der Verehrergemeinde speist. Geoffrey Cubitt und Allen Warren haben in ihrem Sammelband Heroic Reputations and Exemplary Lives (2000) demonstriert, wie die soziale Wirkung und der Einfluss quasi die Schlagkraft - des Heroischen abhängt von der Reputation, dem Ruf, der um heroische Figuren herum konstruiert wird. Diese Konstruktion erfolgt sozial, aber selbstredend, und im 21. Jahrhundert immer stärker und vor allem immer schneller, auch medial. Einen guten Ruf zu erwerben, der normalerweise (aber nicht notwendigerweise) Ehre und einen positiven Leumund einschließt, ist Teil des kulturellen Kapitals um eine Heldenfigur herum. Cubitt und Warren erläutern: „[...] Reputation wird hier nicht als Kondensstreifen eines natürlichen Ruhms verstanden, den die Großen zurücklassen, sondern als kulturelle Konstruktionen, die die Werte und Ideologien der Gesellschaften widerspiegeln, in denen sie produziert werden." 20

Die Fankultur um Lang Lang gehört damit nicht per se zur Figur eines populären Pianisten, sondern sie sagt etwas über die Werte und Vorlieben von globalisierten Gesellschaften im 21. Jahrhundert aus: vielleicht, dass klassische Musik, um viele Menschen zu begeistern, nicht perfekt gespielt sein muss, oder dass der Interpret, um bekannt zu werden, zwangsläufig in möglichst vielen sozialen Netzwerken aktiv und sichtbar sein muss. Etwas Glamour - etwa Lang Langs gutes Aussehen oder seine Vorliebe für modische Kleidung - schadet sicher auch nicht. Dennoch ist Lang Lang keine Persönlichkeit, die ausschließlich für die gute Vermarktung von gutem Aussehen gepaart mit Nichtskönnen berühmt wurde.

Während es auch in früheren Jahrhunderten Celebrities gab, ist die Gegenwart, so Virginia Davis und Barbara Korte, in besonderem Maße gekennzeichnet durch die Tendenz zur Kommodifizierung und Kommerzialisierung von Reputation und ihrer fast ausschließlichen Konstruktion über (Massen-)Medien. Diese Art von Reputation gehört zur Kernbedeutung von Celebrity. Sie ist kurzlebig,

19 Vgl. für celebritas de.langenscheidt.com/latein-deutsch/celebritas und für das Deutsche: Duden. Die deutsche Rechtschreibung, Mannheim 2006.

$20, \ldots[.$.$] reputations are understood here not as the vapour trails of natural glory that the$ great and the good leave behind them, but as cultural constructions reflecting the values and ideologies of the societies in which they are produced." Geoffrey Cubitt / Allen Warren: Introduction: Heroic Reputations and Exemplary Lives, in: dies. (Hg.): Heroic Reputations and Exemplary Lives, Manchester 2000, S. 1-26, hier S. 3. 
nicht notwendigerweise an Werte gebunden und grundsätzlich offen für jeden Menschen, der das Rampenlicht, die Show nicht scheut. ${ }^{21}$

Diese hochgradig mediale Konstruktion von Ruhm, bei der Taten und Werte in den Hintergrund treten, ist im Zeitalter von internetbasierten Massenmedien viel schneller und weiträumiger möglich als noch im ausgehenden 20. Jahrhundert. Das Hauptaugenmerk rückt damit von der Person weg auf die Verehrergemeinde, die bei Celebrities immer wichtiger wird. Mit traditionellem Heldentum hat also Celebrity-Berühmtheit vordergründig wenig zu tun. Die sozialen Prozesse, die zur Herausbildung einer entsprechenden Figur führen, haben jedoch frappierende Ähnlichkeiten. Kristina Sperlich hat gezeigt, dass Heldenstatus und Celebrity-Status sich nicht wechselseitig ausschließen müssen, sondern sich auch verstärken können. „Im Kern der Unterscheidung ist ein moralisches Urteil: Helden werden als moralisch gut und deshalb auch als Rollenvorbilder angesehen. Sie bestätigen den Wertekanon einer Gesellschaft. Celebrities andererseits werden als schlechter Einfluss gesehen, weil sie berühmt sind, ohne notwendigerweise gut zu sein. “22 Es ist aber möglich, zur Celebrity zu werden, ohne den Heldenstatus ernsthaft zu beschädigen; im Gegenteil, ein Ruf als Held kann durch ubiquitäre Präsenz, wie sie eine Celebrity im 21. Jahrhundert hat, weiter erhalten werden. Lang Lang nutzt seinen Status gerade dazu, zu einem Vorbild zu werden, denn er möchte ja Kinder (und Erwachsene) für klassische Musik begeistern und Klavierlernende zum Üben ermutigen. ${ }^{23}$

Dabei hilft ihm sein Ruf der außergewöhnlichen Begabung und seines Könnens, doch seine Reichweite funktioniert über seinen Status als Celebrity. Hinsichtlich von Celebrities und ihrer Funktion innerhalb einer Gesellschaft gibt es eine kulturelle Unsicherheit, weil sie hauptsächlich affektive Reaktionen auslösen, wie etwa Trevor Parry-Giles gezeigt hat. Diese können aber auch positiver Natur sein, etwa wenn eine Celebrity erstrebenswerte Eigenschaften zu vermit-

21 Barbara Korte / Virginia Davis: Editors' Preface, in: The Making of Reputations: Honour Glory - Celebrity. helden. heroes. héros. E-Journal zu Kulturen des Heroischen, Special Issue 2, 2016, S. 5, DOI: 10.6094/helden.heroes.heros./2016/QMR/01: „The present cultural moment, by contrast, seems to be marked by a tendency towards the commodification and total mediatisation of reputation, and its apparent degradation in the form of shallow celebrity - a short-lived, fashionable fame that is seemingly detached from social values and attainable by everybody who is willing to make a spectacle of him- or herself."

Kristina Sperlich: The Hero as Celebrity in Contemporary British Media, in: The Making of Reputations. Honour - Glory - Celebrity. helden. heroes. héros. E-Journal zu Kulturen des Heroischen, Special Issue 2, 2016, S. 81-86, DOI: 10.6094/helden.heroes.heros./ 2016/QMR/13, hier S. 82: „[...] a moral judgement is at the heart of the distinction: heroes are considered as morally good and are therefore also considered as role models. They confirm a society's moral code. Celebrities, on the other hand, are considered as a bad influence because they are famous without necessarily being morally good."

Wie Charisma einer heroisierten Figur zur Motivation von Menschen beiträgt, hat Michael $\mathrm{N}$. Ebertz beschrieben: Charisma und ,das Heroische', in: helden. heroes. héros. E-Journal zu Kulturen des Heroischen 4.2, 2016, S. 5-16, DOI: 10.6094/helden.heroes.heros./2016/ 02/01, hierzu besonders S. 12 . 
teln sucht - wie Lang Lang. ${ }^{24}$ Helden lösen ebenfalls affektive Reaktionen aus, darüber hinaus wird ihnen aber eine besondere symbolische Bedeutung zugeschrieben, die nicht zuletzt mit Ehre in Verbindung gebracht wird, die bei Celebrities eher in den Hintergrund tritt. ${ }^{25}$

Die öffentliche Figur Lang Lang kann hier die Schwächen des Pianisten Lang Lang kompensieren, um erfolgreich im Rampenlicht zu bleiben. Er ist jedenfalls sehr viel mehr als ein einfacher Reality-TV-Star. Der Schluss liegt nahe, dass die deutsche Kritik einen etwas entrückteren Helden und keine Celebrity sehen möchte. Im Fall von Lang Lang drängt sich der Eindruck auf, Gemeinschaften wie die von Musikkritikern würden ihre Wünsche nach Helden auch auf Celebrity-Figuren projizieren. Wie eine Celebrity hat ein Held nicht nur eine Verehrergemeinde, sondern häufig eine Verächtergemeinde, die im Kritisieren genauso viel Verbindendes findet wie die erstere im Verehren und Glorifizieren. Helden wie Celebrities polarisieren gleichermaßen. Der Liszt-Vergleich ist ein Kompliment, denn Liszt ist schließlich als ein Großer mit Fehlern in die Musikgeschichte eingegangen. Helden, so lässt sich immer wieder feststellen, sind Kippfiguren, die für unterschiedliche Gemeinschaften Unterschiedliches aussagen, und an denen sich die Geister scheiden können. Die Feststellung von Achim Aurnhammer und Barbara Korte, dass „,[... ] heroische Figurationen immer auch Komplementärund Gegenvorstellungen [provozieren] und [...] auch in dieser Hinsicht essenziell relationale Kategorien [sind], die für Ambivalenzen und Widersprüche offen sind," lässt sich auch für Celebrities denken. ${ }^{26}$

\section{Beharren jenseits der Krise}

Dass Lang Lang eine Position zwischen Held und Celebrity einnimmt, wird gerade im Moment der Krise besonders deutlich. Denn in Krisen, hier seiner Krankheit, die ihn über einen längeren Zeitraum vom Spielen und vom Konzertieren abhielt, stellt sich die Frage besonders drängend: was bleibt von einem Helden, was von einer Celebrity? Wie lange können beide ,weg vom Fenster sein, aus den Augen der Verehrergemeinde in die Verborgenheit verschwinden, ohne dass der Ruf leidet und der Ruhm aus dem Gedächtnis schwindet? Hier lassen sich Gemeinsamkeiten und Unterschiede der Konzepte gut nachzeichnen. Auch Helden können einfach verschwinden; es bedarf einer Gemeinschaft, die von ihnen erzählt und die Erinnerung an sie lebendig hält. Auch Helden können vergessen werden, wenn sie und die Werte, für die sie stehen, nicht immer wieder aktualisiert werden. Hat eine Person den Status einer Celebrity, scheint ein dauerhaftes Verschwinden wahrscheinlicher, weil eine Celebrity eben im Wesentli-

24 Vgl. Trevor Parry-Giles: Harry Potter and the Paradoxical Critique of Celebrity Culture, in: Celebrity Studies 2.3, 2011, S. 305-319, hier besonders S. 307-308.

25 Vgl. Warren / Cubitt: Heroic Reputations (Anm. 18), S. 3.

26 Aurnhammer / Korte: Einleitung (Anm. 5), S. 13. 
chen von der Verehrergemeinde als einer Art Selbstzweck lebt. Aber eine massenmedial gesteuerte und inszenierte Rückkehr ist möglich, die - im Gegensatz zum Helden - keine spezielle Agenda außer der eigenen Vermarktung haben muss. Die Erregung von Trauer durch einen Helden ist möglich, ja zum Teil, etwa wenn der Held sich opfert, zwingend notwendig, doch durch eine Celebrity eher unwahrscheinlich. Hier ist häufiger Häme zu erwarten, die eine gefallene Lichtgestalt trifft. Entsprechend hat etwa Die Welt im Oktober 2017 puren Spott zu diesem Thema geboten:

Denn es ist eben der linke Arm, welcher dem chinesischen Turbotaster gegenwärtig die Pein einer empfindlich schmerzenden Sehnenscheidenentzündung bereitet. So jedenfalls die offizielle Begründung. Blöde nur für die Deutsche Grammophon [...], weil der stumm geschaltete Klavierspieler eben das gegenwärtig nicht tut: klavierspielend mit Live-Gigs (von Konzerten mag man bei ihm eigentlich nicht mehr sprechen) den Verkauf anstehender CDs und Downloads zu fördern. ${ }^{27}$

Dies ist die Kritik an einer verspotteten Celebrity - alles, was Lang Lang tut, ist Show, und seine Krankheit, so wird insinuiert, vielleicht auch.

Am 1. November 2017 gab Lang Lang Der Zeit ein Interview zu seiner Zwangspause. ${ }^{28}$ Die Journalisten hatten Lang Lang einen Transformer mitgebracht, eine popkulturelle Actionhelden-Figur. Dies war der Anlass, über eine Transformationsphase durch die Krankheit zu sprechen: „Die Entzündung hat mich eine wichtige Lektion gelehrt: Nicht alle Dinge im Leben bekommt man durch Willen und Anstrengung." Lang Lang führte aus, dass Erfolg natürlich nur mit Willen zur Leistung möglich sei, dass aber dieser nicht allein selig macht, und er ließ durchblicken, dass er Veränderungen plane, sobald er seine Gesundheit wiedererlangt habe. „Früher hieß ein erfülltes Leben für mich: jeden zweiten Tag ein Konzert zu spielen. Ich liebe Konzerte, aber ich werde ihre Zahl reduzieren. Es ist besser, ein großartiger Freund zu sein, als einsam an der Spitze zu stehen." Dies ist ein auffallender Entsagungsgestus, der als mögliches Abdanken eines Helden, ein Herabsteigen von seinem Podest gedeutet werden kann. Lang Lang signalisiert hier bereits seinen Plan, sich noch weiter in Richtung Popkultur und Jugendförderung zu entwickeln, den er gegenwärtig einlöst.

Lang Lang hat unzweifelhaft außergewöhnliches Talent und erfüllt damit das Kriterium des Außeralltäglichen und Exzeptionellen. Jeder und jede von uns würde vermutlich gerne auf diesem Niveau ein Musikinstrument spielen können. Er hatte zwar auch Glück, aber es ist offenkundig, dass hinter seinem Erfolg harte Arbeit und leidvolle Lebensphasen stehen. Er hat sich als Vermittler und Verbrei-

27 Manuel Brug: Lang Lang muss wegen Armbeschwerden pausieren, in: Die Welt, 16. Oktober 2017, www.welt.de/print/welt_kompakt_/print_lifestyle/article169665346/Lang-Langmuss-wegen-Armbeschwerden-pausieren.html, 29. Juni 2019.

28

Lang Lang: Ich lerne, geduldig zu sein. Interview mit Manuel J. Hartung und Rudi Novotny, in: Die Zeit, 1. November 2017, www.zeit.de/2017/45/lang-lang-pianist-interview, 30. Juni 2019. 
ter westlicher klassischer Musik weltweit einen Namen gemacht. Damit kann er für ein Leistungs-Heldentum stehen. ${ }^{29}$

Es drängt sich der Verdacht auf, dass Lang Lang zu einer anderen Zeit, ohne die schier erdrückende Wirkung von sozialen Medien, vermutlich nicht so schnell und sicher nicht so sehr im Mainstream bekannt geworden wäre. Lang Lang ist in einer anderen Zeit als dem 21. Jahrhundert schwer vorstellbar, was seine Breitenwirkung angeht. Veronika Zink hat eindrücklich beschrieben, wie uns „die massenmedialen Unterhaltungswelten eine Kulturindustrie des Charismatischen [offerieren], die [...] die Stars, die Ikonen und Kultfiguren an den obersten Rand des Sozialen positioniert" ${ }^{\text {"30 }}$ und wie das Idolatrische nun massenhaft produziert wird. Das scheint ein Widerspruch in sich zu sein, trifft jedoch in den Kern des Problems der verschwimmenden Grenze zwischen Held und Celebrity. Lang Lang ist ein sehr gutes Beispiel dafür, wie sich im 21. Jahrhundert Material, aus dem sich eine Heldenfigur konstruieren lässt, zu etwas anderem, aber dennoch Verwandtem entwickelt. Qua seines musikalischen Talents überschreitet er mit Leichtigkeit kulturelle Grenzen und betont immer wieder das Verbindende an klassischer Musik. Die mediale Präsenz Lang Langs verschmilzt dabei die seriöse Musikerpersönlichkeit mit dem trendbewussten Star, wobei das Star-Sein inzwischen wohl zur Hauptaufgabe geworden ist. Ohne überbordende mediale Dissemination sind Lang Lang, seine Interpretationen klassischer Musik und seine sonstigen Leistungen kaum vorstellbar. Lang Lang kann als Held der Kultur betrachtet werden, der Liszt, Tschaikowski und Rachmaninow zu neuer Wirkung verhilft. Er ist aber auch ein Beispiel für die Flüchtigkeit des Ruhms, eine Berühmtheit mit ubiquitärer Präsenz. Durch seine karitativen Tätigkeiten, unter anderem als UNICEF-Botschafter, knüpft er dennoch gleichzeitig an eine wichtige Facette des Heldentums an und wird potenziell zum moralischen Vorbild, zu einem Helden, der für seine guten Taten bewundert wird, nicht einfach nur für seine Bekanntheit. Das Ausnahmetalent dient erst einmal der Vermarktung und dem zu erzielenden Profit: Hochkultur wird zum Produkt. Diese Wirkung aber wird wiederum zum Ausgangspunkt für soziales Engagement, das weniger weitreichend und effektiv wäre, wenn Lang Lang eben nicht so bekannt wäre. Auffallend ist ein nahezu anti-elitärer Duktus (wobei er von seiner Ausbildung und seiner Position her betrachtet durchaus einer Art Elite angehört): er will kulturelle Teilhabe für möglichst viele Menschen, insbesondere Kinder, erreichen. Dafür nimmt er Abstriche an seiner Reputation in der konventionellen Klassik-Szene in Kauf. Seine Hauptaufgabe im Leben ist also nicht, der beste

29 In der erhellenden Diskussion mit Teilnehmerinnen und Teilnehmern des SISUWorkshops kristallisierte sich die Sichtweise heraus, dass der eigentliche Held hinter der Erfolgsgeschichte Lang Langs Vater sein könnte, der ihn stets zum Üben antrieb. Die VaterSohn-Beziehung war genau deswegen lange Zeit alles andere als unbelastet, wie Lang Lang mehrfach in Interviews berichtet hat.

30 Veronika Zink: Das Spiel der Hingabe. Zur Produktion des Idolatrischen, in: Asch / Butter (Hg.): Bewunderer (Anm. 15), S. 23-43, hier S. 23. 
klassische Pianist zu sein, sondern ein Musikvermittler und Mentor, dem daran gelegen ist, die Freude an der Materie weiterzugeben - so zumindest das Bild, das Lang Lang von sich verbreitet. Dabei sind „Allgegenwärtigkeitsstrategien“, wie Lemke-Mathwey schon 2007 formulierte, ${ }^{31}$ durch Nutzung der Massenmedien unerlässlich.

Lang Lang ist als Fallstudie nicht zuletzt deshalb so aufschlussreich, weil in der deutschen Kritik an ihm häufig andere Bilder von professionellen klassischen Musikern aufgerufen werden, die mehr am Heldisch-Genialischen teilhaben als er selbst. In die Kategorie der Celebrity passt er wiederum nicht perfekt, weil er zu viel Ausnahmetalent mitbringt. In diesem Sinne kann jemand, der seine einmal erschaffene Rolle als glamouröse Celebrity positiv nutzt, durch im weitesten Sinne gute Taten für die Gesellschaft ein Held werden, womit sich ein Kreis schließt.

31 Lemke-Mathwey: Fluch (Anm. 10). 
